\title{
DIFFUSION PROCESSES IN ONE DIMENSION
}

\author{
BY \\ WILLIAM FELLER( $\left.{ }^{1}\right)$
}

1. Introduction. We consider the so-called backward equation of diffusion theory

$$
u_{t}(t, x)=\Omega u(t, x), \quad \Omega=a(x) \frac{d^{2}}{d x^{2}}+b(x) \frac{d}{d x}
$$

in a finite or infinite interval $r_{1}<x<r_{2}$. Recently [2] the theory of semigroups of transformations was used to formulate the integration problem and to define the notion of lateral conditions. It will now be shown that the semi-groups constructed in [2] permit us to describe the totality of all stochastic processes connected with (1.1). To each there corresponds a forward, or Fokker-Planck, equation. It reduces only in simplest cases to the familiar "adjoint"

$$
v_{t}=\Omega^{*} v(t, x), \quad \Omega^{*}=\frac{d}{d x}\left\{a(x) \frac{d}{d x}+b(x) \cdot\right\},
$$

but the general Fokker-Planck equation is not a differential equation.

The present paper consists of two almost independent parts. Its main purpose is a probabilistic discussion of the general diffusion process in one dimension. An informal description of such processes is given in $\$ 2$, which may be read independently of this introduction.

First it is necessary to give a strict definition of the notion of a diffusion process obeying (1.1). This is done in $\$ 3$ in a formal way convenient for the application of semi-group theory. To each diffusion process there corresponds a semi-group of transformations. In [2] we investigated the totality of all such semi-groups transforming functions which are continuous in $\left[r_{1}, r_{2}\right]$ into functions of the same type. In $\$ 4$ it is shown that each such semi-group yields a diffusion process. However, as is shown in \$13, the semi-group associated with a diffusion process may transform a continuous function into a function which is discontinuous at the boundaries. It is for this reason that the semigroups constructed in [2] yield the most general diffusion process in the open interval $\left(r_{1}, r_{2}\right)$, but not in $\left[r_{1}, r_{2}\right]$. For the description of the latter, a rather obvious generalization of the construction of [2] is required.

In $\S 5$ the probabilistic background of diffusion processes is discussed. Prob-

Received by the editors May 9, 1953.

( $\left.{ }^{1}\right)$ Research connected with an Office of Ordnance Research Project at Princeton University, OOR Contract No. DA-36-034-ORD-640. 
ably it soon will be possible to define diffusion processes as Markov processes with continuous path functions. Even the present discussion makes it clear why it is unavoidable to start with the backward equation although the forward equation is intuitively and physically more natural.

These preparations are not required for a formal understanding of the following, and main, part of the paper (at least if manipulations with Laplace transforms are accepted at face value).

In the probabilistic part we make the (unproved but safe) assumption that the path functions $X(t)$ ("the position of the particle at time $t$ ") are continuous except, perhaps, at the boundaries (which may be at infinity $\left({ }^{2}\right)$ ). There exists then a well-defined probability that a path, starting at $x$, will reach a point $\rho_{2}$ without first crossing $\rho_{1}$.

In $\$ 6$ these first passage probabilities are derived from the familiar renewal principle. For points in the interior they are the same for all processes obeying (1.1). In other words, (1.1) determines a class of processes which are not distinguishable as long as no boundary is reached.

Now the interval $\left(r_{1}, r_{2}\right)$ may be finite or infinite, and the diffusion equation (1.1) may be singular in the sense that the coefficients may be unbounded or may vanish near $r_{j}$. Accordingly, the first passage probabilities lead to a classification of accessible and inaccessible boundaries.

With this notion we can proceed to the main purpose of the paper, namely a probabilistic derivation and description of the diffusion processes connected with (1.1). An informal summary is given in the next section.

2. Qualitative description of the processes. In order that there exist one and only one process satisfying (1.1) it is necessary and sufficient that both boundaries be inaccessible; that is, the probability that either boundary be reached within a finite time interval must be zero. A typical example is $u_{t}=u_{x x}$ in $(-\infty,+\infty)$.

If at least one boundary is accessible, then there are infinitely many processes obeying (1.1). A typical example is the case where $r_{j}$ is finite and $a(x)$ and $b(x)$ are continuous at $r_{j}$ with $a\left(r_{j}\right) \neq 0$.

The "absorbing barrier" process is defined by the rule that the process terminates at the moment when a boundary is reached.

Next simplest is the instantaneous return process, which is analogous to a denumerable Markov chain process discussed by Doob [1]. In this process, whenever the boundary $r_{j}$ is reached, an instantaneous return into the interior is effected and the process starts afresh (independently of the past history) from a point $x_{j}$ which is a random variable with distribution function $p_{j}(x)$. In other words, if $X(s) \rightarrow r_{j}$ as $s \rightarrow t-$, then one puts by definition $X(t)=x_{j}$, where

(2) This is done only for the purpose of a probabilistic interpretation of the quantities which appear in the formulas derived in [2]. If this interpretation is granted, the main part of the paper (described in $\$ 2$ ) becomes independent of the preceding. 


$$
\operatorname{Pr}\left\{x_{j} \leqq x\right\}=p_{j}(x),
$$$$
r_{1}<x<r_{2}
$$

The process now starts from scratch.

More generally, there may be a finite sojourn time associated with each boundary, that is, one may have $X(s)=r_{j}$ for a finite time interval $t \leqq s<t+T$. The Markovian character of the process requires that $T$ be a random variable with

$$
\operatorname{Pr}\{T>t\}=\exp \left(-t / \sigma_{j}\right)
$$

where $\sigma_{j}$ is a positive constant. (The limiting cases $\sigma_{j}=0$ and $\sigma_{j}=\infty$ correspond to the instantaneous return process and the absorbing barrier process, respectively.) Moreover, $T$ must be independent of the past. We define now the following set of rules for the

ELEMENTARY RETURN PROCESS. If at time $t$ the boundary $r_{j}$ is reached $\left({ }^{3}\right)$, then $X(s)=r_{j}$ for $t \leqq s<t+T$ where $T$ is a random variable independent of the past and distributed according to (2.2). At time $t+T$ a jump occurs to a point $\mathbf{x}_{j}$ in the interior or to the boundary $\left({ }^{4}\right) r_{k}$ according to the rule

$$
\begin{aligned}
\operatorname{Pr}\left\{X(t+T)=r_{k}\right\} & =p_{j k}, \\
\operatorname{Pr}\left\{r_{1}<X(t+T) \leqq x\right\} & =\tau_{j} p_{j}(x),
\end{aligned}
$$

and the process starts from scratch. Here

$$
p_{j 1}+p_{j 2}+\tau_{j} \leqq 1,
$$

$p_{j k} \geqq 0, \tau_{j} \geqq 0, \sigma_{j} \geqq 0 ;$ and $p_{j}(x)$ are monotonic functions with $p_{j}(x) \rightarrow 0$ as $x \rightarrow r_{1}$ and $p_{j}(x) \rightarrow 1$ as $x \rightarrow r_{2}$.

(The difference between the two sides in (2.4) accounts for the possibility of the process terminating or, in physical language, of masses disappearing.)

It should be noticed that the absorbing barrier process and the instantaneous return process take place on the open interval $\left(r_{1}, r_{2}\right)$; whereas the elementary return process involves the two boundaries as well. (The elastic and reflecting barrier processes below are also processes in $\left(r_{1}, r_{2}\right)$.)

In $\$ 9$ we construct the Laplace transforms of the transition probabilities of such a process from probabilistic considerations. In abstract terminology, they represent the resolvent of a semi-group, and they can be identified with a class of resolvents constructed in [2].

While the elementary return process obeys the backward diffusion equation (1.1), the corresponding Fokker-Planck equation is not (1.2). It has been derived in [2], and in $\$ 10$ it is shown that its obvious physical interpretation

(3) I.e. if $X(s) \rightarrow r_{j}$ as $s \rightarrow t-$ and $r_{1}<X(s)<r_{2}$ for $t-\epsilon<s<t$.

(4) The possibility of a transition from $r_{j}$ to itself is introduced in order to arrive formally at the most general boundary condition of [2]. A simple calculation shows that the same set of rules can be reformulated with $p_{k k}=0$ and a modified mean sojourn time $\sigma_{k}$. The corresponding boundary conditions are analytically equivalent to ours. 
essentially restates our probabilistic definition of the process.

We come next to a class of processes associated in classical diffusion theory with elastic or reflecting barriers. A direct description of the properties of the path functions $X(t)$ of such processes and their behavior near the boundaries is very desirable. It represents an unsolved problem even in the simple case of $u_{t}=u_{x x}$. (The term "reflecting" with its implication of velocities is misleading.)

The elementary return process represents the most general diffusion process in $\left(r_{1}, r_{2}\right)$ except if at least one boundary is regular in the sense of [2]. In this case we obtain analogues to the classical elastic and reflecting barrier processes by a direct passage to the limit from an instantaneous return process. When the boundary $r_{j}$ is reached, with probability $1-\tau_{j}$ we let the process terminate, and with probability $\tau_{j}$ we let it recommence from a point $\rho_{j}$ near $\boldsymbol{r}_{j}$. (In other words, $p_{j}(x)$ has mass one concentrated at $\rho_{j}$.) We now let $\rho_{j} \rightarrow r_{j}$ and at the same time $\tau_{j} \rightarrow 1$ in such a way as to keep certain absorption probabilities constant (cf. §11).

If the boundary $r_{j}$ is regular, this limiting procedure is shown to lead to the class of processes which, in the terminology of [2], depend on regular Green functions. For them, and only for them, does the true Fokker-Planck equation reduce to (1.2). This limiting procedure sheds some new light on the elastic barrier processes, but does not give a measure-theoretic description of the path functions near the boundaries.

For the elastic barrier process one can again define the probabilities that the process terminates at a boundary $r_{j}$, but this event must now be distinguished from the event of reaching the boundary.

The most general diffusion process associated with semi-groups (cf. [2]) is now obtained by starting from an elastic barrier process and superimposing on it the elementary return process in the same way as we have before done with the absorbing barrier process. In other words, when the elastic barrier process in itself would terminate at $r_{j}$, we let the process recommence according to the set of rules described above.

We can calculate the Laplace transforms of all these processes directly. In this way we get a new, and independent, derivation of all the resolvents of the semi-groups constructed in [2]. However, we require the results of [2] for the derivation of the Fokker-Planck equations and of the boundary conditions. Moreover, only the semi-group methods of [2] give us the assurance that we have obtained all diffusion processes of a well-defined class. In particular, the diffusion processes constructed in $\$ 12$ represent the most general type of diffusion process in the open interval $\left(r_{1}, r_{2}\right)$. For the closed interval we have no such complete result, since the associated semi-group may take a continuous function into a discontinuous one. The nature of the most general process appears clear enough ( $\$ 13)$, and explicit formulas for the transition probabilities can easily be calculated by the procedure of $\$ 9$. However, we 
shall not go through the formalities of this derivation nor do we give a formal proof that we are actually dealing with the most general process.

3. Definitions. We shall be concerned with Markovian processes on an interval $E$ which may be finite or infinite, open, half-open, or closed. The end points of $E$ will be denoted by $r_{1}$ and $r_{2}$, where $-\infty \leqq r_{1}<r_{2} \leqq \infty$. Let $X(t)$ represent the path function of the process. We assume that a transition probability

$$
P(t, x ; \tau, \Gamma)=\operatorname{Pr}\{X(\tau) \in \Gamma \mid X(t)=x\}
$$

is defined for all $x \in E$, all Borel sets $\Gamma \subset E$, and $0<t<\tau<\infty$. We do not require that $E$ has assigned probability one, that is, we permit that

$$
P(t, x ; \tau, E) \leqq 1
$$

The difference between the two sides is the probability that the path functions with $X(t)=x$ are defined only in a subinterval of $[t, \tau]$. We assume that the transition probabilities are stationary, that is

$$
P(t, x ; \tau, \Gamma)=P(\tau-t, x, \Gamma),
$$

with $P(t, x, \Gamma)$ defined for all $t>0$.

For simplicity $\left(^{5}\right)$ we introduce the continuity requirement that for each $x \in E$ and each neighborhood $\Gamma$ of $x$

$$
P(t, x, \Gamma) \rightarrow 1,
$$

The Markovian character of the process requires the validity of the Chapman-Kolmogorov identity

$$
P(t+s, x, \Gamma)=\int_{E} P(t, x, d y) P(s, y, \Gamma)
$$

for $t, s>0$. Conversely, suppose that $P(t, y, \Gamma)$ is, for fixed $\Gamma$, Borel measurable in both $t$ and $x$ and, for fixed $(t, x)$, a probability measure on the Borel set of $E$, and also that (3.5) holds. Then $P(t, x, \Gamma)$ can serve as the transition probability of a Markovian process in $\left[r_{1}, r_{2}\right]$. All probability relations in the space of path functions $X(t)$ are then essentially uniquely determined( $\left.{ }^{6}\right)$ by the knowledge of $P(t, x, \Gamma)$ and the initial probability distribution

$$
\mu(\Gamma)=\operatorname{Pr}\{X(0) \in \Gamma\} .
$$

In particular

$$
\operatorname{Pr}\{X(t) \in \Gamma\}=\int_{E} \mu(d x) P(t, x, \Gamma)=V(t, \Gamma) .
$$

(5) For weak continuity properties which always hold cf. [3]. In the present case (3.4) actually follows from the assumed form of the infinitesimal generator.

(6) For the qualification "essentially" cf. Doob, Stochastic processes, New York, Wiley, 1953. 
Note. If $E=\left[r_{1}, r_{2}\right]$ the event $X(t)=r_{j}$ may have a positive probability. Even if $E=\left(r_{1}, r_{2}\right)$, the range of $X(t)$ may coincide with $\left[r_{1}, r_{2}\right]$ but the event $X(t)=r_{j}$ will have probability zero for each fixed $t$. When we refer to a process in the open interval, we refer to the transition probabilities, and only in this wide sense to $X(t)$.

The terminology is not well established, and we shall use the term "process" also to denote the whole class of individual processes with the same transition probabilities $P(t, x, \Gamma)$ and arbitrary initial distributions.

In diffusion theory one usually starts from the assumption that $P(t, x, \Gamma)$ has a probability density $p(t, x, y)$ which for fixed $y$ satisfies the backward equation (1.1). (Cf. §5.) Now the concept of integrating (1.1) as suggested by the semi-group theory leads to a definition of diffusion processes more adapted to our purposes. It is slightly more general, but the main advantage is a considerable simplification of the analytical apparatus.

In effect, the application of semi-group theory to differential equations amounts to a justification of formal operations with Laplace transforms. Classically, the function

$$
u(t, x)=\int_{E} f(y) P(t, x, d y)
$$

is supposed to represent the appropriate solution of (1.1) which reduces to $f(x)$ as $t \rightarrow 0$. Its Laplace transform

$$
F(x)=\int_{0}^{\infty} e^{-\lambda t} u(t, x) d t,
$$

is supposed to be a solution of the ordinary differential equation

$$
\lambda F(x)-\Omega F(x)=f(x),
$$$$
r_{1}<x<r_{2}
$$

In order to avoid further explanations and discussions which are irrelevant to the present paper, we shall start with these properties as our basic definition. This procedure is not elegant, but is simplest for our purposes (and becomes more acceptable when translated into semi-group language).

Definition. A Markovian process on $E$ will be said to obey (1.1) if it possesses transition probabilities satisfying (3.4) and (3.5), and if for every function $f$ which is continuous and bounded on $E$ the Laplace transforms (3.9) satisfy $(3.10)\left({ }^{7}\right)$.

$A$ process will be said to be of the diffusion type if it obeys some equation of the form (1.1).

(7) Note that if $E$ is closed, we require $f$ to be continuous at the end points, but (3.10) puts a restriction only in the interior. This makes it possible that $F(x)$ becomes discontinuous at the end points even if $f$ is continuous. 
(A more satisfactory definition will be proposed in $\$ 5$.)

For simplicity we shall from now on assume that for $r_{1}<x<r_{2}$ the functions $a(x), a^{\prime}(x), b(x)$ are defined and continuous, $a(x)>0$. Note that $a(x)$ and $b(x)$ need not be bounded, and that it is permissible that $a(x) \rightarrow 0$ as $x \rightarrow r_{j}$.

[Note. The preceding definition is sufficient for our purposes, but some readers may wish to see its connection with general semi-group theory and with [2]. To explain this we now anticipate the notations of the following section. The continuity requirement (3.4) implies that for $f \in C$, with the notations of $\$ 4$,

$$
T_{h} f(x) \rightarrow f(x) \quad(h \rightarrow 0+)
$$

for each $x \in\left(r_{1}, r_{2}\right)$ (not necessarily for the boundaries), the convergence being bounded. Unfortunately, in [2] the traditional strong (uniform) continuity was required. Now only the contraction of the semi-group to its range space is strongly continuous, and this circumstance necessitated the clumsy references to the range space in [2]. The above definition would have resulted in greater simplicity and elegance.

With the new continuity requirement it is natural also to modify the usual definition of the infinitesimal generator (for a more general and more systematic approach cf. [3]). If for some $F \in C$ and each $x \in\left(r_{1}, r_{2}\right)$ one has

$$
\frac{T_{h} F-F}{h}(x) \rightarrow \Phi(x)
$$

the convergence being bounded, then we put $\Phi=\bar{\Omega} F$. The operator $\bar{\Omega}$ thus defined will now be called infinitesimal generator of the semi-group. In the Hille-Yosida theory uniform convergence is required, but no difficulties arise. For every $f \in C$ the Laplace transform (3.9) is in the domain of $\bar{\Omega}$ and satisfies

$$
\lambda F-\bar{\Omega} F=f
$$

for given $f$ this equation has only one solution, and the totality of functions for which $\bar{\Omega} F$ is defined is thus identical with the totality of solutions of (3.13).

Our definition of a diffusion process now amounts to requiring that the infinitesimal generator $\bar{\Omega}$ be a contraction of the operator $\Omega$ (that is, if $\bar{\Omega} F$ is defined, then so is $\Omega F$ and $\bar{\Omega} F=\Omega F$, but it is possible that $\Omega F \in C$ without $F$ being of the form (3.9)).

Of course, not every solution of (3.10) defines a semi-group. The totality of solutions with this property has been constructed in [2]; in fact, the arguments of [2] remain valid with the new definition of infinitesimal generator, and the formulations become simpler. However, we can appeal directly to the theorems in [2] as they stand since every semi-group with our continuity 
property is strongly continuous in its range space and thus satisfies the conditions imposed in [2].]

4. Diffusion processes as semi-groups. Let $B$ be the Banach space of bounded Borel measurable functions on $E$ with $\|f\|=\sup |f(x)|$. Let $f \in B$ and consider (3.8). For each fixed $t>0$ we have $u(t, x) \in B$ and $\|u\| \leqq\|f\|$. If we put

$$
u(t, x)=T_{t} f(x)
$$

then $T_{t}$ is a linear operator from $B$ to $B$ which (1) preserves positivity, (2) has norm $\left\|T_{t}\right\| \leqq 1$, and (3) has the semi-group property $T_{t+s}=T_{t} T_{s}$. The last assertion is a direct consequence of (3.5). The three properties are expressed in the statement that the transformations $\left\{T_{t}\right\}$ form a contraction semigroup from $B$ to $B$.

Every Markov process on $E$ thus defines a contraction semi-group from $B$ to $B$. In [2] we have considered such semi-groups subjected to the restriction that they are continuity preserving, that is, that $T_{t} f$ is continuous whenever $f$ is continuous. In this case it is useless to admit discontinuous functions and it is preferable to consider $\left\{T_{t}\right\}$ as a semi-group from $C$ to $C$ where $C$ is the subspace of functions $f$ which are continuous in the closed $\left({ }^{8}\right)$ interval $r_{1} \leqq x \leqq r_{2}$.

We want now to show that each such semi-group defines a Markov process on $\left[r_{1}, r_{2}\right]$.

LEMMA. Let $\left\{T_{t}\right\}$ be a contraction semi-group from $C$ to $C$ such that for each fixed $x$ the function $T_{t} f(x)$ is continuous in $t$. Then for each $t>0$ there exists $a$ kernel $P(t, x, \Gamma)$ which for fixed $x$ is a probability measure on the Borel sets $\Gamma$. For fixed $\Gamma$ this kernel is Borel measurable in $t$ and $x$. Finally

$$
T_{t} f(x)=\int_{r_{1}-}^{r_{2}+} f(y) P(t, x, d y) .
$$

Note that the semi-group property implies the validity of the ChapmanKolmogorov equation (3.5).

Proof. Consider the adjoint semi-group to $T_{t}$ in the following way. If $\mu(\Gamma)$ is any measure on the Borel sets $\Gamma$ in $\left[r_{1}, r_{2}\right]$ the transform $T_{t}^{*} \mu$ is the uniquely defined measure with which

$$
\int_{r_{1-}}^{r_{2}+} T_{t}^{*} \mu(d y) f(y)=\int_{r_{1}-}^{r_{2}+} \mu(d y) T_{t} f(y)
$$

for all $f \in C$. Let $\mu_{x}(\Gamma)$ equal 1 or 0 according as $x$ is, or is not, a point of $\Gamma$. Put

(8) Even if $E$ is open we require that $f(x)$ tends to finite limits as $x \rightarrow r_{j}$. This looks like an essential restriction, but it will be shown in $\$ 13$ that it can be removed and does no harm. 


$$
P(t, x, \Gamma)=T_{t}^{*} \mu_{x}(\Gamma) .
$$

The fact that $T_{t}^{*}$ is positivity preserving and $\left\|T_{t}^{*}\right\| \leqq 1$ implies that $P(t, x, \Gamma)$ is a probability measure for each fixed $t$ and $x$. When the definition (4.4) is inserted in (4.3) one sees that (4.2) holds for all $f \in C$. Finally, if $\Gamma$ is an interval and $\chi_{\Gamma}(x)$ its characteristic function, then there exists a sequence $f_{n} \in C$ such that $f_{n} \downarrow \chi_{\Gamma}$. Then

$$
P(t, x, \Gamma)=\lim _{n \rightarrow \infty} \int_{r_{1-}}^{r_{2}+} P(t, x, d y) f_{n}(y) .
$$

For each $n$ the right side is continuous in $t$ and in $x$, and this guarantees the required measurability properties of $P(t, x, \Gamma)$ for all $\Gamma$.

Now in [2] the equation (3.10) for the Laplace transforms appears as basic requirement for the resolvents. The strong continuity property of the semi-groups used in [2] obviously implies (3.4) and we have thus

ThEOREM 1. Each semi-group from $C$ to $C$ constructed in [2] defines a diffusion process obeying (1.1).

As will be shown in $\$ 13$, the converse is true only for diffusion processes in the open interval $\left(r_{1}, r_{2}\right)$. For a diffusion process in $\left[r_{1}, r_{2}\right]$ it is possible that $f \in C$ but that $u(t, x)$ is not continuous in $\left[r_{1}, r_{2}\right]$.

5. The probabilistic interpretation. As is well known, the backward equation (1.1) has first been derived from probabilistic considerations by Kolmogorov [7]. He showed also that under strong additional conditions the Fokker-Planck equation in the special form (1.2) must hold. Kolmogorov's conditions were relaxed in [4]. The principal step towards generalization was the introduction of the so-called Lindeberg condition which requires that for each fixed $x$ and $\epsilon>0$

$$
\int_{|y-x|>\epsilon} P(t, x, d y)=o(t)
$$

as $t \rightarrow 0$. This, of course, is a strengthening of the continuity condition (3.4).

It is easily seen that a condition of the Lindeberg type is necessary for the continuity of the path functions.

On the other hand, it has been shown recently [5] that under very mild differentiability conditions the Lindeberg condition $\left(^{9}\right)$ alone suffices to derive

( $\left.{ }^{9}\right)$ The abstract formulation is as follows. Let $\left\{T_{t}\right\}$ be a semi-group from $C$ to $C$. The "Lindeberg condition" requires that if $f$ and $g$ agree in a neighborhood of $x_{0}$ then $\left(T_{t}(f-g)\right)\left(x_{0}\right)$ $=o(t), t \rightarrow 0$. Let $\Omega_{0}$ be the infinitesimal generator. The differentiability condition mentioned in the text requires that the Laplace transforms (3.9) be twice differentiable whenever $f^{\prime \prime} \in C$. The theorem states that under these two conditions and if $\left\{T_{t}\right\}$ is a contraction semi-group, $\Omega_{0}$ must be a contraction of an operator $\Omega$ of the form (1.1) with a term $c(x) I$ added-except for the occurrence of singular points where $\Omega$ is of a different form. This theorem characterizes the parabolic differential equations. 
the backward equation (1.1). This is at present the most general derivation of (1.1).

The as yet not completely attained aim of the present method of attack is to define diffusion processes as those Markovian processes for which the path functions $X(t)$ are continuous with probability one as long as they are in the interior of the basic interval $E$ (that is, a discontinuity may occur when a boundary is reached). This definition carries over to locally compact topological spaces.

That all our diffusion processes have this property has apparently never been proved rigorously, but may be assumed with impunity. It is also safe to assume that the continuity of $X(t)$ implies that the corresponding transition probabilities define a contraction semi-group which satisfies the "Lindeberg condition" of the preceding footnote.

This condition expresses essentially that the infinitesimal generator $\Omega_{0}$ is of a local character.

Now the local character of $\Omega_{0}$ and the continuity of $X(t)$ are topological properties, whereas a continuous change of the scale may change differentiable functions into nondifferentiable ones. Hence $\Omega_{0}$ need not be a differential operator. However, the surmise is that in each case the semi-group induces a scale on the $x$-axis such that the functions in the domain $D\left(\Omega_{0}\right)$ become twice differentiable and $\Omega_{0}$ the contraction of a differential operator $\left({ }^{10}\right)$.

Even though the program is as yet unattained, the annoying difference between the forward and backward equations has become understandable. A Markovian process is, essentially, a semi-group in the Banach space of measures. However, not every contraction semi-group on measures defines a Markovian process, whereas each contraction semi-group from $C$ to $C$ does. Only the backward equation refers to semi-groups in $C$ and this explains why only the backward equation can be derived from simple probabilistic conditions.

The corresponding forward equations are much more complicated. They will now be derived probabilistically, but only under the assumption that $X(t)$ is continuous with probability one.

6. First passage times. We consider a diffusion process obeying (1.1) with the coefficients satisfying the conditions stated at the end of $\S 3$. As explained in $\$ 5$ we shall from now on suppose that the path function $X(t)$ is continuous with probability one as long as $r_{1}<X(t)<r_{2}$ (so far no conditions are imposed for the case that a boundary is reached).

Definition. Let $r_{1}<\rho_{1}<\rho_{2}<r_{2}$ be fixed. For any $X(t)$ with $\rho_{1}<X(0)=x<\rho_{2}$ we define the random variable $T_{X}$ as the moment when for the first time $X(t)=\rho_{1}$

${ }^{(10)}$ Essential parts of the proof have been obtained by S. Leader and the author. When proved, the statement will generalize the result described in footnote 9 . It now appears possible to generalize the differential equation (1.1) and to reformulate all the results of [2] in a topologically invariant form. 
or $X(t)=\rho_{2}$. We put

$$
\begin{aligned}
& A\left(t, x ; \rho_{2}, \bar{\rho}_{1}\right)=\operatorname{Pr}\left\{T_{X}<t, X\left(T_{X}\right)=\rho_{2} \mid X(0)=x\right\}, \\
& A\left(t, x ; \rho_{1}, \bar{\rho}_{2}\right)=\operatorname{Pr}\left\{T_{X}<t, X\left(T_{X}\right)=\rho_{1} \mid X(0)=x\right\} .
\end{aligned}
$$

The corresponding Laplace transforms will be called $\xi\left(x ; \rho_{2}, \bar{\rho}_{1}\right)$ and $\xi\left(x ; \rho_{1}, \bar{\rho}_{2}\right)$. Thus

$$
\xi\left(x ; \rho_{2}, \bar{\rho}_{1}\right)=\int_{0}^{\infty} e^{-\lambda t} A\left(t, x ; \rho_{2}, \bar{\rho}_{1}\right) d t \quad(\lambda>0) .
$$

In words, $A\left(t, x ; \rho_{2}, \bar{\rho}_{1}\right)$ is the probability that a path starting from the position $x$ will within time $t$ reach $\rho_{2}$ without previously passing through $\rho_{1}$. Such probabilities occur in the theory of ruin. In physical language $A\left(t, x ; \rho_{2}, \bar{\rho}_{1}\right)$ is the probability that a first passage through $\rho_{2}$ occurs before time $t$ when an absorbing barrier is placed at $\rho_{1}$. Even under the present general conditions one has the familiar

Theorem 2. Putting $z(x)=\xi\left(x ; \rho_{2}, \bar{\rho}_{1}\right)$ one has $\lambda z-\Omega z=0$, and $z(x)$ is the only solution of this equation with $z\left(\rho_{1}\right)=0$ and $z\left(\rho_{2}\right)=\lambda^{-1}$. This solution is monotone.

Before proving this theorem we shall consider some implications. We interpret "the event that the boundary $r_{j}$ has been reached at time $t$ from the interior" (11) as a verbal description of $X(s) \rightarrow r_{j}$ as $s \rightarrow t-0$.

As a corollary of Theorem 2 we shall derive the following results.

THEOREM 3. Let $X(0)=x$, with $r_{1}<x<r_{2}$. The probability that $r_{j}$ will be reached within finite time from the interior is positive if and only if all solutions of $\lambda z-\Omega z=0$ remain bounded as $z \rightarrow r_{j}(\lambda>0)$.

Thus the probability in question is zero for all $x$ or for no $x$. This justifies the

Definition. The boundary $r_{j}$ will be called accessible if and only if there is a positive probability that it will be reached from the interior within a finite time $\left({ }^{12}\right)$ (for all $\left.X(0)=x\right)$.

Thus $r_{j}$ is accessible if and only if all solutions of $\lambda z-\Omega z=0$ are bounded near $r_{j}$. Comparing this with the criteria of $[2, \mathrm{p} .488]$, we see that $r_{j}$ is $a c$ cessible if it is either a regular or an exit boundary.

Theorem 4. Suppose that $r_{2}$ is accessible. Then (a) If $r_{1}$ is inaccessible, there exists one and only one bounded solution $z(x)$ of $\lambda z-\Omega z=0$ with $z(x) \rightarrow \lambda^{-1}$ as $x \rightarrow r_{2}$.

This solution is positive and monotonic. Let $A(t, x)$ be the probability that

(11) Later on we shall consider instantaneous transitions from $r_{j}$ to $r_{k}$.

(12) Cf. footnote 13. 
$r_{2}$ will be reached before time $t$ assuming that the starting position is $X(0)=x$. Then

$$
z(x)=\int_{0}^{\infty} e^{-\lambda t} A(t, x) d t .
$$

(b) If $r_{1}$ is accessible there exists one and only one solution of $\lambda z-\Omega z=0$ with $z(x) \rightarrow 0$ as $x \rightarrow r_{1}$ and $z(x) \rightarrow \lambda^{-1}$ as $x \rightarrow r_{2}$. It is monotonic and for it (6.4) holds provided that $A(t, x)$ is interpreted as the probability that $r_{2}$ will be reached before time $t$ and before $r_{1}$ is reached. (If $r_{1}$ is accessible, then there exist many processes obeying (1.1) with different probabilities of reaching $r_{2}$.)

From Theorem 4 it will be easy to derive the following analogues of classical results concerning probabilities of ruin.

TheOREM 5. Let $r_{2}$ be accessible and let $\Phi(x)=A(\infty, x)$ be the probability of ultimate absorption at $r_{2}$. Then $\Phi(x)$ is the smallest positive solution of $a \Phi^{\prime \prime}(x)$ $+b \Phi^{\prime}=0$ with $\Phi(x) \rightarrow 1$ as $x \rightarrow r_{2}$.

More explicitly: If $r_{1}$ is an accessible boundary, then $\Phi(x)$ is the strictly increasing solution with $\Phi\left(r_{1}\right)=0, \Phi\left(r_{2}\right)=1$. The same holds true whenever

$$
\exp \left\{-\int_{x}^{x_{0}} \frac{b}{a} d s\right\}
$$

is integrable over $\left(r_{1}, x_{0}\right)$. Otherwise $\Phi(x) \equiv 1$. This case arises if $r_{1}$ is an entrance boundary, and may arise if $r_{1}$ is a natural boundary $\left({ }^{13}\right)$.

Note. By the same method one can prove that in the case of two accessible boundaries the expected duration

$$
\psi(x)=\int_{0}^{\infty} t d_{t} A(t, x)
$$

is finite and characterized as the unique solution of $a \psi^{\prime \prime}+b \psi^{\prime}=-1$ with $\psi\left(r_{1}\right)=\psi\left(r_{2}\right)=0$. However, if $r_{1}$ is inaccessible, the boundary condition $\psi\left(r_{1}\right)=0$ can not be imposed, and it can happen that $\psi(x)$ is finite but unbounded, and also that it is infinite for all $x$.

7. Proofs. We start with a few preliminaries. Let

$$
\Pi(x, \Gamma)=\int_{0}^{\infty} e^{-\lambda t} P(t, x, \Gamma) d t, \quad \lambda>0,
$$

and

(13) [Footnote added September 1953.] Doob has discovered the possibility that for a natural boundary $r_{1}$ there may be a positive probability that $X(t) \rightarrow r_{1}$ as $t \rightarrow \infty$ even though $r_{1}$ cannot be reached in finite time. This explains the possibility that $\Phi<1$ even though $r_{1}$ is inaccessible. 


$$
F(x)=\int_{E} f(y) \Pi(x, d y) .
$$

This is defined for all bounded measurable $f$, and agrees with the definition (3.9). Hence, at least when $f$ is continuous, we have by definition

$$
\lambda F-\Omega F=f .
$$

To solve (7.3) for $F$ we require the fact proved by Hille (cf. [2, p. 483]) that there exist two solutions $\xi_{1}$ and $\xi_{2}$ of

$$
\lambda \xi-\Omega \xi=0
$$

satisfying the conditions

$$
\xi_{1}(x) \downarrow, \quad \xi_{2}(x) \uparrow, \quad \xi_{j} \geqq 0 \quad \text { for } r_{1}<x<r_{2} .
$$

In terms of two such solutions we construct the classical Green function

$$
K(x, y)= \begin{cases}\xi_{2}(x) \eta_{1}(y) & \text { for } r_{1}<x \leqq y<r_{2}, \\ \xi_{1}(x) \eta_{2}(y) & \text { for } r_{1}<y \leqq x<r_{2}\end{cases}
$$

where

$$
\eta_{j}(y)=\frac{\xi_{j}(y)}{\xi_{1}(y) \xi_{2}{ }^{\prime}(y)-\xi_{1}{ }^{\prime}(y) \xi_{2}(y)} .
$$

It is known that

$$
F(x)=\int_{r_{1}}^{r_{2}} K(x, y) f(y) d y
$$

is a solution of (7.3). If it is unique, then a comparison of (7.2) and (7.8) shows that

$$
\Pi(x, \Gamma)=\int_{\Gamma} K(x, y) d y .
$$

Now the difference of any two admissible solutions of (7.3) is a bounded solution of (7.4). On the other hand, every solution of (7.4) is a linear combination of $\xi_{1}$ and $\xi_{2}$. As each $\xi_{j}$ is bounded near one boundary, we conclude that (7.4) has a bounded solution only if either $\xi_{1}$ or $\xi_{2}$ is bounded. Independently of the choice of $\xi_{1}$ and $\xi_{2}$ we can therefore state:

(a) If both $\xi_{1}$ and $\xi_{2}$ are unbounded, then the Laplace transform $\Pi(x, \Gamma)$ of the transition probabilities is uniquely determined by (7.3).

(b) If, say, $\xi_{2}$ is bounded but $\xi_{1}$ unbounded, then necessarily

$$
\Pi(x, \Gamma)=\int_{\Gamma} K(x, y) d y+\xi_{2}(x) \Phi_{2}(\Gamma) .
$$


(c) If both $\xi_{1}$ and $\xi_{2}$ are bounded, then

$$
\Pi(x, \Gamma)=\int_{\Gamma} K(x, y) d y+\xi_{1}(x) \Phi_{1}(\Gamma)+\xi_{2}(x) \Phi_{2}(\Gamma) .
$$

Here the $\Phi_{j}(\Gamma)$ are measures on the Borel sets and depend on $\lambda$, but not on $x$. The most general form of these $\Phi_{j}$ was the main topic of [2], but for the present proof we require no special knowledge about $\Phi_{j}$.

Proof of Theorem 2. Let $r_{1}<x<\rho<r_{2}$ and let

$$
H(t, x ; \rho)=\operatorname{Pr}\left\{\operatorname{Max}_{s<t} X(s)>\rho \mid X(0)=x\right\}
$$

be the probability distribution of the first passage time from $x$ to $\rho$. Then, if $\Gamma$ is any set in $\left(\rho, r_{2}\right)$, the event $X(t) \in \Gamma$ can occur only if the first passage has occurred at some time $s<t$. Thus (always assuming the continuity of $X(t))$

$$
P(t, x, \Gamma)=\int_{0}^{t} P(t-s, \rho, \Gamma) d_{s} H(s, x ; \rho) .
$$

For the corresponding Laplace transform

$$
\xi(x ; \rho)=\int_{0}^{\infty} e^{-\lambda t} H(t, x ; \rho) d t \quad \lambda>0,
$$

this means

$$
\Pi(x, \Gamma)=\lambda \Pi(\rho, \Gamma) \xi(x ; \rho) .
$$

Now a glance at (7.9)-(7.11) shows that for any fixed set $\Gamma \subset\left(\rho, r_{2}\right)$ and $x \leqq \rho$ the function $\Pi(x, \Gamma)$ reduces to a linear combination of $\xi_{1}$ and $\xi_{2}$, and hence to a solution of (7.4). Therefore:

$$
\xi(x ; \rho) \text { is a solution of }(7.4) \text { and } \xi(x ; \rho) \rightarrow \lambda^{-1} \text { as } x \rightarrow \rho \text {. }
$$

Note that $\xi(x ; \rho)$ depends on the individual process and cannot be determined without knowing the boundary conditions.

Now let $\rho_{1}<x<\rho_{2}$ and define the first passage transform $\xi\left(x ; \rho_{1}\right)$ in obvious analogy with (7.14). The first passage from $x$ to $\rho_{2}$ can take place either before or after a first passage from $x$ to $\rho_{1}$. This consideration leads to a relation which, translated into the language of Laplace transforms, reads

$$
\xi\left(x ; \rho_{2}\right)=\xi\left(x ; \rho_{2}, \bar{\rho}_{1}\right)+\lambda \xi\left(x ; \rho_{1}, \bar{\rho}_{2}\right) \cdot \xi\left(\rho_{1} ; \rho_{2}\right)
$$

with $\xi\left(x ; \rho_{2}, \bar{\rho}_{1}\right)$ defined in (6.3). Similarly

$$
\xi\left(x ; \rho_{1}\right)=\xi\left(x ; \rho_{1}, \bar{\rho}_{2}\right)+\lambda \xi\left(x ; \rho_{2}, \bar{\rho}_{1}\right) \xi\left(\rho_{2} ; \rho_{1}\right) .
$$

We have here two linear equations for the two unknowns $\xi\left(x ; \rho_{1}, \bar{\rho}_{2}\right)$ and $\xi\left(x ; \rho_{2}, \bar{\rho}_{1}\right)$. From the very definition $(7.14)$ we have $0 \leqq \xi\left(\rho_{i} ; \rho_{j}\right)<\lambda^{-1}$ and therefore the determinant of the system does not vanish. Hence 


$$
\xi\left(x ; \rho_{2}, \bar{\rho}_{1}\right)=\frac{\xi\left(x ; \rho_{2}\right)-\lambda \xi\left(\rho_{1}, \rho_{2}\right) \xi\left(x ; \rho_{1}\right)}{1-\lambda^{2} \xi\left(\rho_{1} ; \rho_{2}\right) \xi\left(\rho_{2} ; \rho_{1}\right)} .
$$

Remembering that $\xi\left(\rho_{j} ; \rho_{j}\right)=\lambda^{-1}$ we find

$$
\lim _{x \rightarrow \rho_{1}} \xi\left(x ; \rho_{2}, \bar{\rho}_{1}\right)=0, \quad \lim _{x \rightarrow \rho_{2}} \xi\left(x ; \rho_{2}, \bar{\rho}_{1}\right)=\lambda^{-1},
$$

and $\xi\left(x ; \rho_{2}, \bar{\rho}_{1}\right)$, being a linear combination of two solutions of $(7.4)$, is itself a solution of (7.4). These conditions determine $\xi\left(x ; \rho_{2}, \bar{\rho}_{1}\right)$ uniquely, and Theorem 2 is proved.

Proof of Theorems 3 and 4. Let $\xi_{j}(x)$ be two solutions of (7.4) satisfying the boundary conditions (7.5). From Theorem 2 we have

$$
\xi\left(x ; \rho_{2}, \bar{\rho}_{1}\right)=\frac{\xi_{2}(x) \xi_{1}\left(\rho_{1}\right)-\xi_{1}(x) \xi_{2}\left(\rho_{1}\right)}{\lambda\left\{\xi_{2}\left(\rho_{2}\right) \xi_{1}\left(\rho_{1}\right)-\xi_{2}\left(\rho_{1}\right) \xi_{1}\left(\rho_{2}\right)\right\}} \quad\left(\rho_{1}<x<\rho_{2}\right) .
$$

Suppose first that $\xi_{1}(x) \uparrow \infty$ as $x \downarrow r_{1}$. Since $\xi_{2}(x)$ is positive and nondecreasing, $\xi_{2}(x)$ remains bounded, and the ratio $\xi_{2}(x) / \xi_{1}(x)$ tends to zero. Hence in this case

$$
\lim _{\rho_{1 \rightarrow r_{1}}} \xi\left(x ; \rho_{2}, \bar{\rho}_{1}\right)=\frac{\xi_{2}(x)}{\lambda \xi_{2}\left(\rho_{2}\right)} .
$$

Next, if $\xi_{1}(x) \uparrow \xi_{1}\left(r_{1}\right)<\infty$ as $x \downarrow r_{1}$, then

$$
\xi^{*}(x)=\xi_{2}(x) \xi_{1}\left(r_{1}\right)-\xi_{1}(x) \xi_{2}\left(r_{1}\right)
$$

is a solution of (7.4) and

$$
\lim _{\rho_{1} \rightarrow r_{1}} \xi\left(x ; \rho_{2}, \bar{\rho}_{1}\right)=\frac{\xi^{*}(x)}{\lambda \xi^{*}\left(\rho_{2}\right)} .
$$

Note that $\xi^{*}(x)$ is obviously nondecreasing, and $\xi^{*}(x) \rightarrow 0$ as $x \rightarrow r_{1}$. Moreover, if $\xi_{2}(x) \uparrow \xi_{2}\left(r_{2}\right)<\infty$ as $x \rightarrow r_{2}$, then $\xi^{*}$ has a finite limit and all solutions of (7.4) remain bounded near $r_{2}$. Hence, as $\rho_{2} \rightarrow r_{2}$, either each of the right-hand members in (7.21) and (7.23) tends to zero, or each tends to a monotone solution of (7.4) which tends to $\lambda^{-1}$ as $x \rightarrow r_{2}$.

Proof of Theorem 5. Put $\xi_{\lambda}(x)=\lambda z(x)$. Then

$$
\xi_{\lambda}(x)=\int_{0}^{\infty} e^{-\lambda t} d_{t} A(t, x)
$$

and hence $\left.{ }^{14}\right) \xi_{\lambda}(x) \uparrow \Phi(x)$ as $\lambda \downarrow 0$. Solving the differential equation $\Omega u=f$ and substituting $u=f=\xi_{\lambda}$ gives an integral equation for $\xi_{\lambda}$ which shows that

(14) Alternatively, this can be seen directly from $\lambda\left(\xi_{\lambda}-\xi_{\nu}\right)-\Omega\left(\xi_{\lambda}-\xi_{\nu}\right)=(\nu-\lambda) \xi_{\nu}(\nu>\lambda)$ which shows (since $\xi_{\nu}>0$ ) that $\xi_{\lambda}-\xi_{\nu}$ can have no negative minimum. If $r_{1}$ is accessible, then $\xi_{\lambda}-\xi_{\nu}$ vanishes at both ends. If $r_{1}$ is inaccessible the result follows by approximation. 
$\xi_{\lambda} \uparrow \Phi$ implies that $\Omega \Phi$ exists and $\Omega \Phi=\lim \Omega \xi_{\lambda}=\lim \lambda \xi_{\lambda}=0$. Clearly $\Phi\left(r_{2}\right)=1$ since $\xi_{\lambda}(x) \uparrow 1$ as $x \rightarrow r_{2}$. Suppose now that the integral (6.5) diverges as $x \rightarrow r_{1}$. Then $\Phi \equiv 1$ is the only solution of $\Omega \Phi=0$ with $\Phi\left(r_{2}\right)=1$. On the other hand, if the integral (6.5) converges, there exists exactly one solution of $\Omega u=0$ with $u\left(r_{1}\right)=0, u\left(r_{2}\right)=1$. From $\Omega\left(u-\xi_{\lambda}\right)=-\lambda \xi_{\lambda}<0$ it is seen that the function $u-\xi_{\lambda}$ can have no minimum. Since it vanishes at $x=r_{1}, r_{2}$ it follows that $\xi_{\lambda}(x) \leqq u(x)$, whence $\Phi(x) \leqq u(x)$. The inequality would require $\Phi$ to assume negative values, and this proves the theorem.

8. The absorbing barrier process. Consider a process obeying (1.1) and assume, as before, that the path functions $X(t)$ are continuous.

DeFINITION. Using the random variable $T_{X}$ of the definition of $\$ 6$ we define the absorbing barrier process $\left({ }^{15}\right)$ corresponding to our process and $\left(\rho_{1}, \rho_{2}\right)$ as the process of the path functions $X_{\mathrm{abs}}(t)$ defined by

$$
X_{\text {abs }}(t)=\left\{\begin{array}{l}
X(t) \\
X\left(T_{X}\right)
\end{array}\right.
$$

$$
\begin{aligned}
& \text { for } t \leqq T_{X}, \\
& \text { for } t \geqq T_{X}
\end{aligned}
$$

with the obvious probability relations induced by the original process.

We shall refer to $T_{X}$ as the absorption time. With the notation of $\S 6$ we have

$$
\operatorname{Pr}\left\{T_{X} \leqq t\right\}=A\left(t, x ; \rho_{1}, \bar{\rho}_{2}\right)+A\left(t, x ; \rho_{2}, \bar{\rho}_{1}\right) .
$$

TheOREM 6(16). Let $P_{\mathrm{abs}}(t, x, \Gamma)$ be the transition probability of the absorbing barrier process and $\Pi_{\mathrm{abs}}(x, \Gamma)$ its Laplace transform (7.1). With the notations of $\$ 6$ we have $\left.{ }^{17}\right)$ for $r_{1}<\rho_{1}<x<\rho_{2}<r_{2}$

$$
\begin{aligned}
& \Pi_{\mathrm{abs}}\left(x, \rho_{1}\right)=\xi\left(x ; \rho_{1}, \bar{\rho}_{2}\right), \\
& \Pi_{\mathrm{abs}}\left(x, \rho_{2}\right)=\xi\left(x ; \rho_{2}, \bar{\rho}_{1}\right) .
\end{aligned}
$$

For any Borel set $\Gamma \subset\left(\rho_{1}, \rho_{2}\right)$ we have

$$
\Pi_{\mathrm{abs}}(x, \Gamma)=\int_{\mathbf{\Gamma}} K(x, y) d y
$$

where $K(x, y)$ is the Green function ( $c f$. (7.6)) of $\lambda u-\Omega u=f$ formed by means of two solutions $\xi_{1}$ and $\xi_{2}$ of $\lambda \xi-\Omega \xi=0$ satisfying the boundary conditions

$$
\xi_{1}\left(\rho_{1}\right)=0, \quad \xi_{2}\left(\rho_{2}\right)=0 .
$$

Proof. Clearly, (8.3) is only a restatement of Theorem 2. From this

(15) By (8.1) the absorbing barrier process is defined in the closed interval $\left[\rho_{1}, \rho_{2}\right]$. If we let $X_{\mathrm{abs}}(t)$ be undefined for $t \geqq T_{X}$ we get the corresponding process for the open interval.

(16) This theorem restates a well known fact. Also the renewal method for the proof is familiar. Both theorem and proof are (under slightly different hypotheses) found in a recent paper by Darling and Siegert [0]. (This paper reached the author after completion of the present manuscript.)

${ }^{(17)}$ In (8.3) and similar formulas later on the letters $\rho$ or $r$ are used not only to denote a number, but also for the set consisting of the single point $\rho$ or $r$. 
theorem one has also that for $\rho_{1}<x<y<\rho_{2}$

$$
\xi\left(x ; y, \bar{\rho}_{1}\right)=\frac{\xi_{1}(x)}{\lambda \xi_{1}(y)} .
$$

Again, the renewal argument used in (7.15) shows that for $\Gamma \subset\left(y, \rho_{2}\right)$ one has

$$
\begin{aligned}
\Pi_{\mathrm{abs}}(x, \Gamma) & =\lambda \xi\left(x ; y, \bar{\rho}_{1}\right) \Pi_{\mathrm{abs}}(y, \Gamma) \\
& =\frac{\xi_{1}(x)}{\xi_{1}(y)} \Pi_{\mathrm{abs}}(y, \Gamma) .
\end{aligned}
$$

Thus

$$
\Pi_{\mathrm{abs}}(x, \Gamma)=\xi_{1}(x) \cdot F(\Gamma)
$$

where $F(\Gamma)$ is independent of $\lambda$. This relation holds only for sets $\Gamma \subset\left(x, \rho_{2}\right)$, but a similar relation holds for sets $\Gamma \subset\left(\rho_{1}, x\right)$. The theorem now follows on comparing this result with the general form of the transition probabilities given in (7.11).

DEFINITION. We shall say the transition probabilities of a process are defined by $a$ Green function if for $r_{1}<x<r_{2}$ and $\Gamma \subset\left(r_{1}, r_{2}\right)$ the corresponding Laplace transform $\Pi(x, \Gamma)$ is of the form (8.4) where $K(x, y)$ is a regular Green func$\operatorname{tion}\left({ }^{18}\right)$.

Note that no restriction is placed on the transitions $\Pi\left(x, \rho_{j}\right)$ and $\Pi\left(\rho_{j}, \Gamma\right)$.

TheOREM 7. (a) If both $r_{1}$ and $r_{2}$ are inaccessible, then as $\rho_{1} \rightarrow r_{1}$ and $\rho_{2} \rightarrow r_{2}$ the transition probabilities of the absorbing barrier process for $\left(\rho_{1}, \rho_{2}\right)$ converge to transition probabilities in $\left(r_{1}, r_{2}\right)$ which are defined by the unique regular Green function for $\left(r_{1}, r_{2}\right)$. In this case there exists one and only one process in $\left(r_{1}, r_{2}\right)$ obeying (1.1).

(b) If $r_{1}$ is inaccessible but $r_{2}$ accessible, convergence takes place to transition probabilities defined by that regular Green function which is formed by means of a solution with $\xi(x) \downarrow 0$ as $x \rightarrow r_{2}$.

(c) If $r_{1}$ and $r_{2}$ are accessible, then limiting transition probabilities exist and are defined by that regular Green function which is formed by means of solutions $\xi_{j}(x)$ with $\xi_{j}(x) \downarrow 0$ as $x \rightarrow r_{j}$.

This theorem extends the notion of absorbing barrier processes to intervals $\left(r_{1}, r_{2}\right)$ with accessible boundaries.

Proof. In the proof of Theorem 3 (\$7) it has been shown that as $\rho_{1} \rightarrow r_{1}$ and $\rho_{2} \rightarrow r_{2}$ the solutions $\xi\left(x ; \rho_{1}, \bar{\rho}_{2}\right)$ and $\xi\left(x ; \rho_{2}, \bar{\rho}_{1}\right)$ tend to certain limits. Using these limiting forms the theorem becomes trivial.

It follows in particular that whenever both $r_{1}$ and $r_{2}$ are accessible there exists

(18) Cf. (7.6). The notion of regular Green function was introduced in [2] and refers to Green functions formed by means of solutions satisfying the monotonicity conditions (7.5). The restriction to regular Green functions is essential for the validity of some of our statements. 
an absorbing barrier process in $\left(r_{1}, r_{2}\right)$ obeying (1.1).

The relevant statements concerning the boundary conditions and the Fokker-Planck equations are contained in Theorem 10, as the limiting case $c_{i}=0$.

Note on the corresponding densities. It would be pleasing to assert that the transition probabilities of the last theorem are determined by densities $p(t, x, y)$, and that the latter satisfy both the backward equation

$$
p_{t}(t, x, y)=a(x) p_{x x}(t, x, y)+b(x) p_{x}(t, x, y)
$$

and the forward equation

$$
p_{t}(t, x, y)=[a(y) p(t, x, y)]_{y y}-[b(y) p(t, x, y)]_{y} .
$$

When both boundaries are inaccessible, $p(t, x, y)$ should be uniquely determined by either of these equations; if $r_{j}$ is accessible, one should expect that for (8.9) the boundary condition $p(t, x, y) \rightarrow 0$ as $x \rightarrow r_{j}$ must be imposed; in the case of (8.10) the corresponding boundary condition is

$$
a(y) \exp \left[-\int b(y) a^{-1}(y) d y\right] p(t, x, y) \rightarrow 0
$$

as $y \rightarrow r_{j}$.

These assertions are contained in known results about differential equations provided that both boundaries are regular and that more stringent conditions on $a(x)$ and $b(x)$ are imposed. Unfortunately, the theory of semigroups leads directly only to a slightly weaker result. We should prove that for fixed $x, y$

$$
K(x, y)=\int_{0}^{\infty} e^{-\lambda t} p(t, x, y) d t
$$

where $p(t, x, y)$ has a bounded derivative with respect to $t$. Actually it appears that without deeper analysis one can assert only that $\left({ }^{19}\right)$

$$
K(x, y)=\int_{0}^{\infty} e^{-\lambda t} V(d t, x, y)
$$

where $V$ is a nondecreasing function of $t$.

Now we have seen that for $x<z<y$

$$
K(x, y)=\frac{\xi(x)}{\xi(z)} K(z, y) .
$$

Here the second factor on the right is of the form (8.13), while the first

(19) (8.13) is a direct consequence of the Bernstein-Hausdorff-Widder theorem (cf. [8]) and the well known property of resolvents according to which $(-1)^{n} d^{n} K(x, y) / d \lambda^{n}=n$ ! $\cdot K_{n+1}(x, y) \geqq 0$ where $K_{n}$ denotes the iterated kernels; cf. [6, p. 99]. 
factor is the Laplace transform of the first passage time from $x$ through $z$. But the convolution of two monotonic functions will be continuous, or differentiable, whenever one of the two components has this property. It follows therefore that if $p(t, z, y)$ is, for a fixed $z, y$, a continuous (differentiable) function of $t$, then the same is true for any $x, z$. Moreover, (8.14) shows that for the density $p(t, x, y)$ to satisfy (8.9) it is necessary and sufficient that the first passage time density be a solution of the backward equation (1.1). The duality principle of [2] permits us to translate these assertions into corresponding statements referring to $p(t, x, y)$ as function of $t$ and $y$, and to (8.10) instead of (8.9).

Accordingly, to prove the opening surmise of this note it would suffice to prove that for fixed $x<z$ the ratio $\xi(x) / \xi(z)$ is the Laplace transform of a differentiable function or, alternatively, that this is true of $K(x, x)$. It seems plausible that this is provable by the methods of the general theory. Unfortunately, the author sees at present only a method of proof which is based on laborious estimates. This does not seem natural, in particular since under slightly stronger conditions the facts are known. Moreover, the assertion would improve only the aesthetical appeal and simplify some formulations, without being essential.

9. The elementary return process. We now proceed to calculate the transition probabilities of the process defined in $\$ 2$. For definiteness we assume for the time being that both boundaries are accessible, and $\sigma_{1}>0, \sigma_{2}>0$. The starting position $X(0)=x$ is assumed to be the interior $\left(r_{1}, r_{2}\right)$.

(a) The probability that up to time $t$ no transitions from a boundary have occurred and that $X(t)=r_{1}$ (i.e. the first boundary to be reached is $r_{1}$, and the first sojourn time has not expired) is

$$
\int_{0}^{t} d_{8} A\left(t-s, x ; r_{1}, \bar{r}_{2}\right) \cdot e^{-s / \sigma_{1}}
$$

where $A\left(t, x ; r_{1}, \bar{r}_{2}\right)$ is the distribution function of the absorption time defined in (6.1). For the corresponding Laplace transforms (6.3) we introduce the notations

$$
\xi_{1}(x)=\xi\left(x ; r_{1}, \bar{r}_{2}\right), \quad \xi_{2}(x)=\xi\left(x ; r_{2}, \bar{r}_{1}\right) .
$$

Then (9.1) has the Laplace transform

$$
\lambda \xi_{1}(x) \frac{\sigma_{1}}{1+\lambda \sigma_{1}}
$$

(b) At the expiration of the sojourn time a "jump" occurs. The probabilities that it takes $X(t)$ to the interior, to $r_{1}$, or to $r_{2}$, are $\tau_{1}, p_{11}, p_{12}$, respectively. The probability density that this jump occurs at time $t$ is the expression (9.1) multiplied by $\sigma_{1}^{-1}$. 
(c) Let $P^{(n)}(t, x, \Gamma)$ be the probability that up to time $t$ exactly $n$ "jumps" occur and that $X(t) \in \Gamma$. Let $\Pi^{(n)}(x, \Gamma)$ be'the corresponding Laplace transform. Then

$$
\Pi^{(0)}(x, \Gamma)=\Pi_{\mathrm{abs}}(x, \Gamma),
$$

provided $\Gamma$ is a set in the interior. If $\Gamma$ reduces to a boundary point then, as shown under (a),

$$
\Pi^{(0)}\left(x, r_{j}\right)=\lambda \xi_{j}(x) \frac{\sigma_{j}}{1+\lambda \sigma_{j}} .
$$

(d) The probability density that the $n$th jump occurs at $r_{j}$ and at time $t$ is $P^{(n-1)}\left(t, x, r_{j}\right) \cdot \sigma_{j}^{-1}$. It has the Laplace transform

$$
\Pi^{(n-1)}\left(x, r_{j}\right) \sigma_{j}^{-1} \text {. }
$$

(e) If the $n$th jump occurs at $r_{j}$ at time $s$, then for every set $\Gamma \subset\left(r_{1}, r_{2}\right)$

$$
\operatorname{Pr}\{X(s+) \in \Gamma\}=\tau_{j} \int_{\Gamma} d p_{j}(y) .
$$

Therefore the probability that $X(t+s) \in \Gamma$ and that no further absorption has taken place is

$$
\tau_{j} \int_{r_{1}}^{r_{2}} P_{\mathrm{abs}}(t, y, \Gamma) \cdot d p_{j}(y) .
$$

For the corresponding Laplace transform we introduce the abbreviation $\left({ }^{20}\right)$

$$
U_{j}(\Gamma)=\tau_{j} \int_{r_{1}}^{r_{2}} \Pi_{\mathrm{abs}}(y, \Gamma) d p_{j}(y) .
$$

Thus for $\Gamma \subset\left(r_{1}, r_{2}\right)$

$$
\Pi^{(n)}(x, \Gamma)=\sum_{j=1}^{2} \Pi^{(n-1)}\left(x, r_{j}\right) \sigma_{j}^{-1} U_{j}(\Gamma) .
$$

(f) If at any time a jump takes place at $r_{i}$, the probability density that it leads into the interior and then, after a time $t$, to a first absorption at $r_{j}$ has the Laplace transform

$$
\lambda \tau_{i j}=\lambda \tau_{i} \int_{r_{1}}^{r_{2}} \xi_{j}(x) d p_{i}(x) .
$$

(g) From (d) and (f) we conclude

$$
\Pi^{(n)}\left(x, r_{j}\right)=\sum_{i=1}^{2} \Pi^{(n-1)}\left(x, r_{i}\right) \sigma_{i}^{-1}\left\{p_{i j}+\lambda \tau_{i j}\right\} \frac{\sigma_{j}}{1+\lambda \sigma_{j}} .
$$

${ }^{(20)}$ In comparing our formulas with those of [2], put $U_{j}(\Gamma)=\int_{\Gamma} Q_{j}(x) d x$ where $Q_{j}(x)$ is the density defined in (21.14). 
For convenience of writing we introduce the matrix notation

$$
p=\left(p_{i j}\right), \quad \tau=\left(\tau_{i j}\right), \quad \boldsymbol{o}=\left(\begin{array}{ll}
\sigma_{1} & 0 \\
0 & \sigma_{2}
\end{array}\right) .
$$

For row and column matrices we write

$$
\left[x_{i}\right]={ }_{x_{2}}^{x_{1}}, \quad\left[x_{i}\right]^{\prime}=x_{1} x_{2} .
$$

Now (9.12) takes on the form

$$
\left[\Pi^{(n)}\left(x, r_{j}\right)\right]^{\prime}=\left[\Pi^{(n-1)}\left(x, r_{i}\right)\right]^{\prime} \boldsymbol{\delta}^{-1}\{p+\lambda \tau\}\{I+\lambda \boldsymbol{\delta}\}^{-1} \boldsymbol{\delta}
$$

where $I$ is the identity matrix. By definition we have

$$
\Pi(x, \Gamma)=\sum_{n=0}^{\infty} \Pi^{(n)}(x, \Gamma) .
$$

Thus, using (9.5) and (9.15), we get

$$
\begin{aligned}
{\left[\Pi\left(x, r_{j}\right)\right]^{\prime} } & =\lambda\left[\xi_{j}\right]^{\prime}(I+\lambda \boldsymbol{\delta})^{-1} \sum_{n=0}^{\infty}\left(\{p+\lambda \tau\}\{I+\lambda \boldsymbol{\delta}\}^{-1}\right)^{n} \boldsymbol{\delta} \\
& =\lambda\left[\xi_{j}\right]^{\prime}(I+\lambda \boldsymbol{\delta})^{-1}\left(I-\left\{p+\lambda_{\tau}\right\}\{I+\lambda \boldsymbol{\delta}\}^{-1}\right)^{-1} \boldsymbol{\delta}
\end{aligned}
$$

For abbreviation put $\left({ }^{21}\right)$

$$
R=(I+\lambda \delta-p-\lambda \tau)^{-1} .
$$

Then $(9.17)$ reduces to

$$
\left[\Pi\left(x, r_{j}\right)\right]^{\prime}=\lambda\left[\xi_{j}\right]^{\prime} \boldsymbol{R} \boldsymbol{\delta} .
$$

Having thus $\Pi\left(x, r_{j}\right)$ we get $\Pi(x, \Gamma)$ from (9.16) and (9.10). For $\Gamma \subset\left(r_{1}, r_{2}\right)$ we get

$$
\Pi(x, \Gamma)=\Pi_{\mathrm{abs}}(x, \Gamma)+\lambda\left[\xi_{j}\right]^{\prime} R\left[U_{j}(\Gamma)\right] .
$$

Finally, for completeness we give the corresponding expressions for the case where the process starts at a boundary. The argument which led to (9.19) shows that

$$
\left(\Pi\left(r_{i}, r_{j}\right)\right)=R \boldsymbol{\delta}
$$

and this is also evident from (9.19). Similarly, for $\Gamma \subset\left(r_{1}, r_{2}\right)$

$$
\left[\Pi\left(r_{i}, \Gamma\right)\right]=R\left[U_{i}(\Gamma)\right],
$$

as is again clear from (9.20).

(21) The proof that $R$ exists is given in [2, p. 512]. It should have been mentioned there that the proof would formally break down if $\pi_{i}=\sigma_{i}=\tau_{i}=0$ and $p_{i i}=1$. However, that case is impossible since it would reduce a lateral condition to $0=0$. 
In (9.19)-(9.22) we have now expressions for all the transition probabilities. The very derivation shows that they have the necessary properties like positivity and that they determine a process obeying (1.1).

We observe that our process is a special case of the one treated in $[2, \S 21]$. If one puts there $\pi_{1}=\pi_{2}=0$, the formulas agree with ours. The new derivation is more direct, but we still must refer to [2] for the derivation of the lateral condition and the proof that they determine the process.

Summing up we have

THEOREM 8. If both boundaries are accessible, then there exists exactly one diffusion process in $\left[r_{1}, r_{2}\right]$ obeying (1.1) and having the properties of the "elementary return process" described in \$2. If, and only if, $\sigma_{1}=\sigma_{2}=0$ the process takes place in the open interval $\left(r_{1}, r_{2}\right)$. The solutions of (1.1) corresponding to our process are characterized by the two lateral conditions

$$
\begin{aligned}
\left(1-p_{11}\right) u\left(t, r_{1}\right)-p_{12} u\left(t, r_{2}\right) & =\tau_{1} \int_{r_{1}}^{r_{2}} u(t, y) d p_{1}(y)-\sigma_{1} \Omega u\left(t, r_{1}\right), \\
-p_{21} u\left(t, r_{1}\right)+\left(1-p_{22}\right) u\left(t, r_{2}\right) & =\tau_{2} \int_{r_{1}}^{r_{2}} u(t, y) d p_{2}(y)-\sigma_{2} \Omega u\left(t, r_{2}\right) .
\end{aligned}
$$

Note. For lucidity of exposition we have been assuming that both boundaries are accessible. The starting point was the absorbing barrier process defined in the open interval $\left(r_{1}, r_{2}\right)$. The new process plays in the closed interval $\left[r_{1}, r_{2}\right]$, unless either $\sigma_{1}$ or $\sigma_{2}$ vanishes (in which case the corresponding boundary is excluded).

If both $r_{1}$ and $r_{2}$ are inaccessible, then no return process is possible. If $r_{1}$ is inaccessible and $r_{2}$ accessible, the above construction applies except that no returns can occur from $r_{1}$.

10. The corresponding Fokker-Planck equations. If $\mu(\Gamma)$ is the probability distribution for the initial point $X(0)$, then the distribution of the position $X(t)$ is given by (3.7). Consider now in particular the transition probabilities of the elementary return process determined by (9.20). The set functions $U_{j}(\Gamma)$ are defined in (9.8) and (9.9). As explained in $\$ 8$, the distribution with the Laplace transform $U_{j}(\Gamma)$ has a density, and the same is then true of our transition probability. We can therefore write

$$
\begin{aligned}
V(t, \Gamma) & =\int_{\Gamma} v(t, y) d y, & \Gamma \subset\left(r_{1}, r_{2}\right), \\
V\left(t, r_{j}\right) & =V_{j}(t) . &
\end{aligned}
$$

In $\$ \$ 18$ and 22 of [2] we have studied, in the Banach space of measures, the semi-group which is the adjoint to the semi-group from $C$ to $C$ corresponding to the process of $\S 9$. The following theorem is a restatement of 
these results in terms of differential equations $\left({ }^{22}\right)$.

TheOREM 9. (a) In the process of Theorem 8 let the mean sojourn times $\sigma_{j}$ be positive. Then

$$
a(x) v_{x}(t, x)-b(x) v(t, x)=\Phi_{v}(t, x)
$$

exists and is of bounded variation. For every set $\Gamma \subset\left(r_{1}, r_{2}\right)$ one has $\left({ }^{23}\right)$

$$
\frac{\partial}{\partial t} \int_{\Gamma} v(t, x) d x=\int_{\Gamma} d_{x}\left\{\Phi_{v}(t, x)+\frac{\tau_{1}}{\sigma_{1}} V_{1}(t) p_{1}(x)+\frac{\tau_{2}}{\sigma_{2}} V_{2}(t) p_{2}(x)\right\}
$$

and

$$
\begin{aligned}
& V_{1}^{\prime}(t)=-\frac{1-p_{11}}{\sigma_{1}} V_{1}(t)+\frac{p_{21}}{\sigma_{2}} V_{2}(t)+\Phi_{v}\left(t, r_{1}\right), \\
& V_{2}^{\prime}(t)=\frac{p_{12}}{\sigma_{1}} V_{1}(t)-\frac{1-p_{22}}{\sigma_{2}} V_{2}(t)-\Phi_{v}\left(t, r_{2}\right)
\end{aligned}
$$

where

$$
\Phi_{v}\left(t, r_{j}\right)=\lim _{x \rightarrow r_{j}} \Phi_{v}(t, x) .
$$

(b) If $\sigma_{1}=\sigma_{2}=0$ put $V_{1}(t)=V_{2}(t)=0$ and (10.4) is to be replaced $\left({ }^{24}\right)$ by

$$
\frac{\partial}{\partial t} \int_{\Gamma} v(t, x) d x=\int_{\Gamma} d_{x}\left\{\Phi_{v}(t, x)+\tau_{1} \Phi_{v}\left(t, r_{1}\right) p_{1}(x)-\tau_{2} \Phi_{v}\left(t, r_{2}\right) p_{2}(x)\right\} .
$$

(c) These equations are the Fokker-Planck (or forward) equations of our process. Depending on the nature of the boundaries $\left({ }^{25}\right) r_{j}$, they either determine the process uniquely, or one or two boundary conditions of the form (8.11) are satisfied and characterize the process.

Physical interpretation. Let $\Gamma=\left(\rho_{1}, \rho_{2}\right)$ be an interval contained in $\left(r_{1}, r_{2}\right)$.

${ }^{(22)}$ In order not to obscure a simple situation, the formulation of the theorem avoids an explicit reference to the fact (described at the end of \$8) that the existence and the necessary regularity properties of the density $v(t, x)$ have been proved in [2] explicitly only for the case when the initial distribution $\mu(\Gamma)$ is sufficiently smooth (that is, belongs to the range of the resolvent). For an arbitrary initial distribution the theorem has been completely proved only under slightly more stringent conditions on the coefficients $a(x)$ and $b(x)$.

${ }^{(23)}$ Note that in the case $\tau_{1}=\tau_{2}=0$ equation (10.4) reduces to the classical differential equation (1.2). If $p_{1}(x)$ and $p_{2}(x)$ are differentiable, the integrals in (10.4) may be omitted and we still have a differential equation.

(24) Similarly, if $\sigma_{1}=0$ and $\sigma_{2}>0$ the term $\sigma_{1}^{-1} V_{1}(t)$ in (10.4) and (10.6) is to be replaced by $\Phi_{v}\left(t, r_{1}\right)$.

${ }^{(25)}$ No lateral conditions are required if $r_{j}$ are "exit" boundaries. If $r_{j}$ is a "regular" boundary, a lateral condition is required. In $[2, \S 22]$ both boundaries are supposed to be regular. The modification for exit boundaries is contained in $\$ 16$ of [2]. 
The left side of (10.4) represents the rate of change of the mass in $\Gamma$. On the right the term $\Phi_{v}\left(t, \rho_{2}\right)-\Phi_{v}\left(t, \rho_{1}\right)$ represents the net flux through the boundaries into $\Gamma$. The term $\tau_{j} V_{j}(t)\left\{p\left(\rho_{2}\right)-p\left(\rho_{1}\right)\right\} \sigma_{j}^{-1}$ expresses that a direct flow takes place into $\Gamma$ from the boundary $r_{j}$. The flow of mass $V_{j}(t)$ at the boundaries is described as follows. The boundary $r_{1}$ loses mass at the rate $\left(1-p_{11}\right) \sigma_{1}^{-1}$ times the instantaneous mass (exponential decay). Of this mass the proportion $p_{12} /\left(1-p_{11}\right)$ flows to $r_{2}$, and $\tau_{1} /\left(1-p_{11}\right)$ into the interior. On the other hand, $r_{1}$ gains mass at a rate $p_{21} \sigma_{2}^{-1} V_{2}(t)$ from $r_{2}$, and at a rate $\Phi_{v}\left(t, r_{1}\right)$ through a continuous influx from the interior. For our particular process this gain can be shown to be positive. Thus the Fokker-Planck equations give a restatement in physical terms of the original probabilistic description of the process in $\S 2$.

The case $\sigma_{j}=0$ means that no mass can accumulate at $r_{j}$ and the return to the interior, if it takes place, is instantaneous. Therefore the flow into $\Gamma$ is no longer proportional to $V_{j}(t)$ but to the instantaneous flux at the boundary.

\section{The elastic barrier process.}

DeFinition. The boundary $r_{j}$ is regular if the functions

$$
W(x)=\exp \left\{-\int^{x} b(s) a^{-1}(s) d s\right\} \quad \text { and } \frac{1}{a(x) W(x)}
$$

are integrable in some (and therefore every) neighborhood of $r_{j}$.

According to [2, p. 516] every regular boundary is accessible, but an "exit" boundary is accessible without being regular. For the understanding it is preferable to express the regularity as a condition on the functions $\xi_{j}$ and $\eta_{j}$ of (7.5) and (7.7) by means of which the Green function is formed: $r_{j}$ is regular if and only if $\xi_{j}(x)$ is bounded and $\eta_{j}(y)$ is integrable in $\left(r_{1}, r_{2}\right)$.

Our purpose is now to derive the classical elastic barrier process by a passage to the limit from a diffusion process in $\left(r_{1}, r_{2}\right)$, obeying (1.1). This process is defined as the following modification of the absorbing barrier process:

(11.2) When the boundary $r_{j}$ is reached there is probability $\tau_{j} \geqq 0$ of an instantaneous transfer to the point $\rho_{j}$ in the interior, and probability $1-\tau_{j}$ that the process terminates.

In the return process thus described let $\gamma_{j}$ be the probability that a path starting at $\rho_{j}$ will be absorbed at $r_{j}$.

For simplicity, we shall assume that both boundaries are regular and shall study the following

Passage to the limit. We let $\rho_{j} \rightarrow r_{j}$ and at the same time $\tau_{j} \rightarrow 1$ in such a way that the probability $\gamma_{j}<1$ remains constant $\left.{ }^{26}\right)$. (There is no harm in putting, say, $\tau_{1}=0$ and letting only $\tau_{2} \rightarrow 1$.)

${ }^{(28)}$ The same result would be obtained if instead of $\gamma_{j}$ one keeps constant the expected duration of the time to the ultimate absorption at $r_{j}$. 
(a) We start by calculating $\gamma_{j}$. Put

$$
F(x)=\int_{r_{1}}^{x} W(s) d s .
$$

Then $a F^{\prime \prime}+b F^{\prime}=0$ and by Theorem 5 the probability, in the absorbing barrier process, that a path starting at $\rho_{2}$ will terminate at $r_{2}$ is $F\left(\rho_{2}\right) / F\left(r_{2}\right)$. Accordingly

$$
\gamma_{2}=\frac{F\left(\rho_{2}\right)}{F\left(r_{2}\right)}\left(1-\tau_{2}\right) \sum_{n=0}^{\infty}\left\{\tau_{2} \frac{F\left(\rho_{2}\right)}{F\left(r_{2}\right)}\right\}^{n} .
$$

For abbreviation put

$$
\theta_{2}=\left\{F\left(r_{2}\right)-F\left(\rho_{2}\right)\right\} / F\left(r_{2}\right) .
$$

Then from (11.4)

$$
1-\tau_{2}=\frac{\gamma_{2}}{1-\gamma_{2}} \frac{F\left(r_{2}\right)}{F\left(\rho_{2}\right)} \cdot \theta_{2}
$$

and thus

$$
\lim _{\rho_{2} \rightarrow \tau_{2}} \frac{1-\tau_{2}}{\theta_{2}}=\frac{\gamma_{2}}{1-\gamma_{2}}=c_{2} .
$$

A similar calculation applies to $r_{1}$.

(b) In the notations of $\$ 9$ our return process is characterized by $\sigma_{j}=0$, $p_{i j}=0$, while $p_{j}(x)$ is a unitary distribution function with the jump at $\rho_{j}$. The process is restricted to the interior of $\left(r_{1}, r_{2}\right)$ and its transition probabilities are given in (9.20). In the present case we have

$$
R=(I-\lambda \tau)^{-1}
$$

where the matrix $\tau$ is defined (cf. (9.11)) by

$$
\tau_{i j}=\tau_{i} \xi_{j}\left(\rho_{i}\right) .
$$

The set function $U_{j}(\Gamma)$ is defined in (9.9). If $\Gamma \subset\left(\rho_{1}, \rho_{2}\right)$ it is easily seen that

$$
U_{1}(\Gamma)=\tau_{1} \xi_{2}\left(\rho_{1}\right) H_{1}(\Gamma), \quad U_{2}(\Gamma)=\tau_{2} \xi_{1}\left(\rho_{2}\right) H_{2}(\Gamma)
$$

with

$$
H_{j}(\Gamma)=\int_{\mathbf{\Gamma}} \eta_{i}(y) d y .
$$

Since $\rho_{j} \rightarrow r_{j}$, it is clear that it suffices to consider sets $\Gamma \subset\left(\rho_{1}, \rho_{2}\right)$ so that we may use (11.10). We now proceed to investigate the asymptotic behavior of (11.9) and (11.10). 
(c) We recall that $\xi_{2}(x)$ is a nondecreasing solution of $\lambda \xi-\Omega \xi=0$ with $\xi\left(r_{1}\right)=0, \xi\left(r_{2}\right)=\lambda^{-1}$. From [2] we know (formula (20.2) after eliminating the restriction $a(x) \equiv 1)$ that for regular boundaries the quantities

$$
\omega_{i j}=(-1)^{i} \lim _{x \rightarrow r_{j}} W^{-1}(x) \xi_{i}^{\prime}(x)
$$

exist, and form a symmetric, nonsingular matrix $\omega$.

Now, for example,

$$
\begin{aligned}
1-\tau_{22} & =1-\tau_{2} \lambda \xi_{2}\left(\rho_{2}\right)=1-\tau_{2}+\tau_{2} \lambda \int_{\rho_{2}}^{r_{2}} \xi_{2}^{\prime}(x) d x \\
& =1-\tau_{2}+\dot{\lambda} \tau_{2} \int_{\rho_{2}}^{r_{2}}\left\{\omega_{22}+o(1)\right\} W(x) d x .
\end{aligned}
$$

Using (11.5) and (11.7) we get from this

$$
\frac{1-\tau_{22}}{\theta_{2}} \rightarrow c_{2}+\lambda \omega_{22}
$$

To simplify the writing put

$$
\boldsymbol{\theta}=\left(\begin{array}{ll}
\theta_{1} & 0 \\
0 & \theta_{2}
\end{array}\right), \quad \mathrm{c}=\left(\begin{array}{ll}
c_{1} & 0 \\
0 & c_{2}
\end{array}\right) .
$$

Then (11.13) and its analogues can be combined into the matrix equation

$$
\theta^{-1}(I-\tau) \rightarrow c+\lambda \omega \text {. }
$$

The same calculation applied to (11.10) yields

$$
\theta_{j}^{-1} U_{j}(\Gamma) \rightarrow-\omega_{12} H_{j}(\Gamma) .
$$

Hence

$$
R\left[U_{j}\right]=(I-\tau)^{-1} \boldsymbol{\theta} \theta^{-1}\left[U_{j}\right] \rightarrow-(c+\lambda \omega)^{-1}\left[H_{j}(\Gamma)\right] \omega_{12}
$$

Thus

$$
\Pi(x, \Gamma) \rightarrow \Pi_{\mathrm{abs}}(x, \Gamma)-\omega_{12}\left[\xi_{j}\right]^{\prime}(\mathbf{c}+\lambda \omega)^{-1}\left[H_{j}(\Gamma)\right] .
$$

Now the right side can be obtained from the resolvent (21.12) of [2] by the specialization $\sigma_{i}=\sigma_{j}=0,\left(1-\pi_{i}\right) \pi_{i}^{-1}=c_{i}$. (It should be noted that our solutions $\eta_{j}(x)$ in terms of which $H_{j}(\Gamma)$ is defined in (11.10a) differ from the corresponding solutions $\eta_{j}(x)$ introduced in [2, formula (20.3)] by a factor $-\omega_{12}{ }^{-1}$ as can be seen by comparing the Green function (20.9) of [2] and (7.6) above.) We therefore need no further verification that the right side of (11.18) really represents transition probabilities. 
This proves that part of the following theorem which refers to the backward equation (1.1). The statements concerning the Fokker-Planck equation are a consequence of Theorem 9. We have thus

TheOREM 10. Our limiting process leads to a diffusion process in the open interval $\left(r_{1}, r_{2}\right)$ which obeys (1.1). The corresponding Fokker-Planck equation is given by (1.2). The solutions of (1.1) are in this case characterized by the lateral conditions

and those of (1.2) by

$$
c_{i} u(t, x)=(-1)^{i-1} \lim _{x \rightarrow r_{i}} W^{-1}(x) u_{x}(t, x)
$$

$$
c_{i} \lim _{x \rightarrow r_{i}} W(x) a(x) v(t, x)=(-1)^{i} \Phi_{v}\left(t, x_{i}\right)
$$

(cf. (10.3) and (11.1)).

For $c_{i}=0$ the boundary $r_{i}$ becomes "reflecting," for $c_{i} \rightarrow \infty$ the process reverts into the absorbing barrier process. (The limiting boundary conditions remain correct.)

It is clear that the resulting formulas do not depend on the assumption that both boundaries are regular: one elastic barrier can be combined with any other type at the other end. However, if $r_{1}$ is inaccessible, then the probability of absorption at $r_{2}$ may be identically one for all $\rho$, and the mean duration of the absorbing barrier process may be infinite for all $\rho$. In this case, therefore, one needs a new interpretation for $\gamma_{i}$, but one may get it trivially by a new passage to the limit.

12. The general process induced by semi-groups in $C\left[r_{1}, r_{2}\right]$. The elastic barrier process takes place in the open interval $E=\left(r_{1}, r_{2}\right)$. One has $P(t, x, E) \rightarrow 0$ as $t \rightarrow \infty$, except when both boundaries are reflecting $\left(c_{j}=0\right)$, or when one boundary is inaccessible and the other reflecting. If $P(t, x, E) \rightarrow 0$ there is probability one that the process terminates either at $r_{1}$ or $r_{2}$, and we speak again of an "absorption." Note, however, that the boundary $r_{j}$ can be reached without an absorption taking place. In fact, the process can start from a boundary $\left({ }^{27}\right)$.

It is now possible to superimpose the elementary return process described in $\$ 2$ to any elastic barrier process in the same way as we have superimposed it to the absorbing barrier process. An alternative way, requiring no new calculations, consists in a direct passage to the limit. For that purpose we replace (11.2) by the following conditions.

${ }^{(27)}$ Let $\xi_{i}^{*}(x)$ denote the Laplace transform of the probability that an absorption occurs at $r_{j}$ before time $t$, given that $X(0)=x$. If both boundaries are regular, it can be shown without difficulties that the $\xi_{i}^{*}(x)$ are solutions of $\lambda \xi-\Omega \xi=0$ satisfying the four boundary conditions $\quad \xi_{1}^{*}\left(r_{1}\right)+\xi_{2}^{*}\left(r_{1}\right)=\xi_{1}^{*}\left(r_{2}\right)+\xi_{2}^{*}\left(r_{2}\right)=\lambda^{-1}, \quad c_{1} \xi_{2}^{*}\left(r_{1}\right)-\lim _{x \rightarrow r_{1}} \quad W^{-1}(x) \xi_{2}^{* \prime}(x)=0, \quad c_{2} \xi_{1}^{*}\left(r_{2}\right)$ $+\lim _{x \rightarrow r_{2}} W^{-1}(x) \xi_{1}^{* \prime}(x)=0$. In particular, $\xi_{2}^{*}\left(r_{1}+\right)$ is the (positive) probability of an absorption at $r_{2}$ given that the process starts at $r_{1}$. 
(12.1) Consider an elementary return process in which $r_{i}$ has a mean sojourn time $\epsilon_{i} \sigma_{i}$. When a jump from $r_{i}$ occurs, it leads with probability $\epsilon_{i} p_{i j}$ to $r_{j}$, with probability $1-\epsilon_{i}$ to the point $\rho_{i}$, and with probability $\epsilon_{i} \tau_{i}$ to a random point of $\left(r_{1}, r_{2}\right)$ with probability distribution $p_{i}(x)$. Here $p_{i 1}+p_{i 2}+\tau_{i} \leqq 1$. We effect the same passage to the limit $\epsilon_{i} \rightarrow 0, \rho_{i} \rightarrow r_{i}$ as described in $\$ 11$.

It is not necessary to repeat the calculations since a simple inspection shows that the only change is that the term $c_{i}$ is replaced by another expression which equally remains unaffected by the passage to the limit. Assuming both boundaries to be regular, the procedure of $\$ 11$ shows that the transition probabilities of the limiting process are determined by

$$
\Pi(x, \Gamma)=\Pi_{\mathrm{abs}}(x, \Gamma)-\omega_{12} \lambda\left[\xi_{j}(x)\right]^{\prime} R\left\{\left[c_{i}^{-1} H_{i}(\Gamma)\right]+\left[U_{i}(\Gamma)\right]\right\}
$$

with $U_{i}(\Gamma)$ defined in $(9.9), H_{i}(\Gamma)$ in (11.10a), and $\left({ }^{28}\right)$

$$
R=\left\{I-p+\lambda \delta-\lambda \tau+\lambda c^{-1} \omega\right\}^{-1} .
$$

Furthermore

$$
\begin{aligned}
{\left[\Pi\left(x, r_{i}\right)\right]^{\prime} } & =-\omega_{i 2} \lambda\left[\xi_{j}(x)\right]^{\prime} \boldsymbol{R \delta}, \\
{\left[\Pi\left(r_{i}, \Gamma\right)\right] } & =-\omega_{i 2} R\left\{\left[c^{-1} H_{i}(\Gamma)\right]+\left[U_{i}(\Gamma)\right]\right\}, \\
\Pi\left(r_{i}, r_{k}\right) & =-\omega_{12} R \mathbf{R} .
\end{aligned}
$$

If only $r_{2}$ is regular, $c_{1}^{-1}$ must be replaced by 0 , and if $r_{1}$ is inaccessible, one has to put $p_{11}=p_{12}=\tau_{1}=\sigma_{1}=0$.

It has been shown in [2] that in the case $\sigma_{j} \neq 0$ the Fokker-Planck equation is still given by (10.4)-(10.6) but that the boundary conditions

$$
c_{j} V_{j}(t)=\sigma_{j} \lim _{x \rightarrow r_{j}} \Phi_{v}(t, x)
$$

must be added. On the other hand, the lateral conditions (9.23) for the backward equation are changed by the addition of the term $-c_{i} \lim (-1)^{i} W^{-1}(x) u_{x}(t, x)$ to the right-hand member of the $i$ th equation.

\section{The most general process.}

THEOREM 11. The process with transition probabilities (12.2)-(12.6) represents the most general diffusion process in $\left[r_{1}, r_{2}\right]$ with the property that the associated semi-group (4.2) transforms each $f \in C\left[r_{1}, r_{2}\right]$ into a function which is again continuous in $\left[r_{1}, r_{2}\right]$. However, there exist diffusion processes without the last property.

Proof. The first assertion can be verified by comparing the formulas

${ }^{28}$ In trying to verify that this result agrees with the equivalent for the resolvent given in (22.2) of [2] one should put $c_{i}=\left(1-\pi_{i}\right) / \pi_{i}$ and observe the following change in notations: The quantities $p_{i j}, \tau_{i}, \sigma_{i}$ of $[2]$ correspond to $p_{i j}\left(1-\pi_{i}\right), \tau_{i}\left(1-\pi_{i}\right)$, and $\sigma_{i}\left(1-\pi_{i}\right)$ in the present notations. Furthermore, the solutions $\eta_{i}(x)$ of $[2]$ are the same as $-\omega_{12} \eta_{j}(x)$ in the present notation. 
(12.3)-(12.6) with the resolvent of the most general contraction semi-group from $C$ to $C$ given in $[2,(21.12)]$. To substantiate the last assertion consider the

EXAMPLE. Let $\mathrm{P}_{\mathrm{abs}}(t, x, \Gamma)$ be the transition probability of the process corresponding to $u_{t}=u_{x x}$ in $E=(0, \infty)$ with an absorbing barrier at $x=0$. We now stipulate that if $x=0$ is reached at time $t$, then with probability $1 / 2$ we shall have $X(\tau)=0$ for $\tau \geqq t$, while with probability $1 / 2$ the process terminates. In other words, we put

$$
\begin{array}{ll}
P(t, x, \Gamma)=P_{\text {abs }}(t, x, \Gamma) & \text { for } \Gamma \subset(0, \infty), \\
P(t, 0, \Gamma)=0, & \\
P(t, x, 0)=(1 / 2)\left\{1-P_{\text {abs }}(t, x, E)\right\}, & P(t, 0,0)=1 .
\end{array}
$$

Then

$$
\begin{array}{rlrl}
u(t, x) & =\int_{0+}^{\infty} P_{\mathrm{abs}}(t, x, d y) f(y)+(1 / 2)\left\{1-P_{\mathrm{abs}}(t, x, E)\right\} f(0) \\
& \rightarrow(1 / 2) f(0) & \text { as } x \rightarrow 0+
\end{array}
$$

while $u(t, 0)=f(0)$.

THEOREM 12. The transition probabilities of the most general diffusion process in the open interval $\left(r_{1}, r_{2}\right)$ are given by (12.2) with $\sigma_{1}=\sigma_{2}=0$. The corresponding Fokker-Planck equation is (10.8), subjected to the lateral conditions

$$
\left[\psi_{j}\right]^{\prime} \mathbf{c}(I-p)+\left[(-1)^{i} \Phi_{v}\left(t, r_{j}\right)\right]^{\prime}=0
$$

where $\Phi_{v}$ is defined in (10.3) and

$$
\psi_{i}=\lim _{x \rightarrow r_{i}} W(x) a(x) v(t, x)
$$

with $W(x)$ given in (11.1).

The interesting feature is that although the boundaries $r_{j}$ are excluded, the lateral conditions (13.1) include the terms $p_{i j}$ which correspond to passages from $\left({ }^{29}\right) r_{i}$ to $r_{j}$.

Proof. We have seen that the Laplace transform $F$ of (4.1) satisfies (7.3). If $f$ is continuous in $\left(r_{1}, r_{2}\right)$ and if $f(x)$ approaches limits as $x \rightarrow r_{j}$, then the same is true of $F$. This follows [2, Theorem 13.1] and the fact that the bounded solutions of $\lambda \xi-\Omega \xi=0$ (if they exist) approach limits as $x \rightarrow r_{j}$. The Hille-Yosida theorem now implies that our semi-group takes $C$ into $C$. The most general diffusion process in $\left(r_{1}, r_{2}\right)$ is therefore among those constructed in $\$ 12$, and it is obvious that $\sigma_{1}=\sigma_{2}=0$ is necessary and sufficient for the

(29) They account for events such as $X(t) \rightarrow r_{1}$ as $t \rightarrow t_{0}-$ and $X(t) \rightarrow r_{2}$ as $t \rightarrow t_{0}+$, with $X\left(t_{0}\right)$ undefined. 
process to be restricted to the open interval. The lateral condition (13.1) is not explicitly derived in [2], but is obtained by a calculation analogous to that given on p. 514 .

To return to diffusion processes in the closed interval, let $C^{*}$ denote the subspace of $B$ consisting of those $f(x)\left(r_{1} \leqq x \leqq r_{2}\right)$ which are continuous for $r_{1}<x<r_{2}$ and approach finite limits as $x \rightarrow r_{j}$ (these limits need not coincide with $f\left(r_{j}\right)$ ). Using the definition of the infinitesimal generator proposed in [3] it is easily seen that for a process obeying (1.1) the semi-group (4.1) transforms $C^{*}$ into itself, that is, that $f \in C$ implies $T_{t} f \in C$. Using the methods of [2], it is possible to construct the totality of contraction semi-groups from $C^{*}$ to $C^{*}$ and then to choose among them those which correspond to a stochastic process (that is, where the adjoint semi-group transforms measures into measures).

It is to be expected that the most general diffusion process in $\left[r_{1}, r_{2}\right]$ is obtained by superimposing an elementary return process to any process in $\left(r_{1}, r_{2}\right)$ in which there is a positive probability (and therefore certainty) that the process will terminate after a finite time $\left({ }^{30}\right)$. The existence of such processes can be proved, and their transition probabilities can be obtained, by a straightforward generalization of the arguments of $\$ 9$. However, the proof that this leads to the most general diffusion process seems to require a lengthy argument.

\section{REFERENCES( $\left.{ }^{31}\right)$}

0. D. A. Darling and A. J. F. Siegert, The first passage problem for a continuous Markoff process, Ann. Math. Statist. vol. 24 (1953) pp. 624-639.

1. J. L. Doob, Markoff chains-Denumerable case, Trans. Amer. Math. Soc. vol. 58 (1945) pp. $455-473$.

2. W. Feller, The parabolic differential equations and the associated semi-groups of transformations, Ann. of Math. vol. 55 (1952) pp. 468-519. (1953).

3. - Semigroups of transformations in general weak topologies, Ann. of Math. vol. 57

4. —, Zur Theorie der stochastischen Prozesse, Math. Ann. vol. 113 (1936) pp. 113-160.

5. - On positivity preserving semi-groups of transformations on $C\left[r_{1}, r_{2}\right]$, Annales Société Mathématique Pologne.

$\left.{ }^{(30}\right)$ Note that in such a process four things may happen when $X(t) \rightarrow r_{i}$. (1) The process may terminate, (2) $X(t)$ may continue to vary continuously in $r_{1}<x<r_{2}$ as in the elastic barrier process, (3) $X(t)$ may instantaneously change to a random point in the interior, (4) $X(t)$ may for a time remain constant, $X(t)=r_{i}$, and then change to a random point in the interior. Thus there are two types of jumps into the interior. The condition that the associated semi-group $T_{t}$ changes $C$ into $C$ makes it impossible that both types occur in the same process from the same boundary. The general lateral condition contains four functions $p_{i}(x)$ of bounded variation.

(31) [September 1953] I am obliged to J. L. Doob for a reference to W. Doeblin, Sur certains mouvements saleatoires, C. R. Acad. Sci. Paris vol. 208 (1939) pp. 249-250. In this note our criteria for accessibility are announced. Doeblin's method seems to be based on a direct study of the path functions and the law of the iterated logarithm. It appears that the proof has never been published. 
6. E. Hille, Functional analysis and semi-groups, Amer. Math. Soc. Colloquium Publications, vol. 31, New York, 1948.

7. A. Kolmogorov, Ueber die analytischen Methoden in der Wahrscheinlichkeitsrechnung, Math. Ann. vol. 104 (1931) pp. 415-458.

8. D. V. Widder, The Laplace transform, Princeton University Press, 1941.

Princeton University,

Princeton, N. J.

STOCKHOLM UNIVERSITY,

STOCKHOLM, SWEdEN. 